\title{
Téoros
}

Revue de recherche en tourisme

\section{Recherche et banque de données touristiques}

\section{René Baretje}

Volume 13, numéro 1, mars 1994

URI : https://id.erudit.org/iderudit/1077778ar

DOI : https://doi.org/10.7202/1077778ar

Aller au sommaire du numéro

Éditeur(s)

Université du Québec à Montréal

ISSN

0712-8657 (imprimé)

1923-2705 (numérique)

Découvrir la revue

Citer cette note

Baretje, R. (1994). Recherche et banque de données touristiques. Téoros, 13(1), 49-49. https://doi.org/10.7202/1077778ar d'utilisation que vous pouvez consulter en ligne.

https://apropos.erudit.org/fr/usagers/politique-dutilisation/ 


\section{Recherche et banque de données touristiques}

Note de M. René Baretje, Directeur du Centre des hautes études touristiques, Aix-en-Provence, France

Le tourisme met en jeu, soit sur un plan national, soit sur un plan international, des opérations de capital, des opérations de production et des opérations de consommation effectuées conjointement ou successivement par l'État, les institutions financières. les entreprises et les ménages, dans un environnement où les dimensions politique. sociale, culturelle, écologique sont loin d'être absente.

Résoudre les problèmes de planification, d'aménagement, de prévision, de financement, de construction, de production, de gestion ${ }_{r}$ de commercialisation, de promotion et de publicite, de formation, de transport, etc., exige alors une approche pluridisciplinaire et mondiale, dès lors que la consommation touristique n'est qu'une consommation différée dans le temps et dans l"espace.

Qui plus est, tout pays, quel que soit son niveau de développement, est dépendant à un moment donné de l"étranger sur le plan des capitaux, des services, des biens d'équipement et de consommation, de la main d'oeuvre. II se doit, dans ce contexte, de connaitre et de respecter les règles qui régissent les échanges internationaux, et ceci est particulièrement manifeste en matiére touristique.

Nul pays, en outre, même s'il croit posséder la meilleure industrie touristique, ne peut rester indifférent aux réalisations étrangères. II doit donc se tenir régulièrement informé des innovations administratives, juridiques, techniques, économiques, financières, sociales, fiscales, etc. s' $s^{\prime} i l$ ne veut pas ètre rejoint, voire dépassé.

Bref, I'homme contemporain vit environ 640 000 heures; il en consacre 60000 au travail et 280000 aux loisirs. Demain, avec la réduction de la joumée, de la semaine, de l'année et de la vie de travail il ne travaillera que 40 000 heures et disposera de 300000 heures de loisir. Sa propension croissante a consommer de la distance, à accroittre son rayon d'action, à diversifier ses produits, donc ses destinations, en fait un grand devoreur d'espaces qu'il faut aménager, exploiter, gérer, protéger, dans son intérêt comme dans celui de la collectivité toute entière.

Ce nomade privilégié des temps modernes. à la recherche de la récréation, sera trés bientôt survi ou imité par une masse impor= tante de l'humanité quil ne bénéficie pas encore des bienfaits de la civilisation dite de loisirs.

Résoudre les problèmes d'aujourd'hui et préparer la solution de ceux de demain exigent donc une mobilisation immédiate de tous les centres de décision au niveau local, régional, national, intracontinental, intercontinental. Toutes les forces vives des secteurs public et privé contribuant à la détente, à la distraction et au développement de l'homme sont concernées, du politique à l'aménageur, du sociologue au médecin, du juriste au financier, de l'architecte à l'économiste, de l'ingénieur au futurologue, de l'anthropologue à l'informaticien, du géographe au psychologue, etc, aucune profession ne peut être exclue, directement ou indirectement, du circuit production = consommateur. Tout le monde participe de près ou de loin à la grande fète planétaire, soit pour la promouvoir, soit pour l'organiser, soit pour l'arienter, soit pour la réglementer le cas échéant. Pour faire de cette fête - les vacances - une réussite, encore faut-il savoir gérer à bon escient le temps, le revenu et l'espace, trilogie de base de toute politique intégrée en matière touristique.

Pour faciliter la tâche des chercheurs et décideurs privés ou publics, le Centre des Hautes Etudes Touristiques de l'Université de Droit, d'Economie et des Sciences d'AixMarseille a réuni au fil des ans le plus important fonds documentaire au monde, couvrant les multiples facettes du tourisme et des loisirs de plein air Isituation au 1 er juillet 1993,51300 liwres + environ 7000 articles de revues scientifiques).

Cent soixante tomes de Bibliographie Touristique (Collection aÉtudes et Mémoiresw) classent par theme et par pays toute la littérature réunie dans la bibliothèque d'Aixen-Provence. Chaque mois environ, sort un nouveau tome contenant 1500 références nouvelles.

Le Centre des Hautes Etudes Touristiques n'a pas la prétention; niles movens, de réunir tous les travaux parus à travers le monde sur le tourisme et les loisirs. C'est la raison pour laquelleil a créé, il y a une douzaine d'années. une nouvelle collection intitulee $w$ Essaism, qui réunit d'une manière exhaustive les références de toute la litterature internationale connue du CHET et concernant unecentaine de thèmes spécifiques (ex: écotourisme / multiplicateur / balance des paiements / carrying capacity, etc.) et une vingtaine de destinations touristiques présentant un intérêt particulier lex.; Chine, Japon, Mexique, etc.). Six cents volumes (chacun contenant 250 nouvelles références) sont d'ores et dejà disponibles. Cette collection est appelée à s'enrichir chaque année d'une cinquantaine de nouveaux titres.

Pour accélérer le traitement de la documentation. le Centre a mis au point un programme informatique qui, à partir d'un thesaurus (evolutif) de 1300 mots clé et d'un classement géographique allant du niveau du monde jusqu'au niveau de la commune, permet de connaittre dans des délais extrèmement brefs les références de travaux concernant tel(s) theme(s) et/ou tel(s) pays. L'analyse de contenu des 45000 derniers documents est d'ores et déjả en mémoire d'ordinateurpermettant à la w Touriscothèque Informatiséen d'être opérationnelle. Elle signale parexemple l'existence de 532 travaux sur le multiplicateur touristique, de 97 documents sur la perception de l'image d'une destination, de 80 travaux sur le tourisme à Fidil, de 134 documents sur la Chine ou encore de 493 travaux sur le tourisme du troisième age.

Certes, au fil des mois, ce service deviendra de plus en plus performant. Mais les personnes ou institutions de l'industrie touristique, concernées par une approche pluridisciplinaire et mondiale du phénomène du tou= risme et des loisirs, peuvent dorénavant s'adresser directement au CHET:

Centre des Hautes Etudes Touristiques Fondation Vasarely

1. Avenue Marcel Pagnol

13090 Aix-en-Provence

Tel.: (33) 42200973

Fax: (33) 42205098

Pour obtenir des listings d'ordinateur (1 référence $=1$ page) par thème et/ou index géographique. Le tarif de la consultation est fixé sur la base suivante:

$50 \mathrm{FF}$ (soit environ $11,30 \$ \mathrm{can}$ ) de prlse en charge + 5 FF (environ 1.15 \$ can) par référence bibliographique.

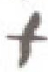

